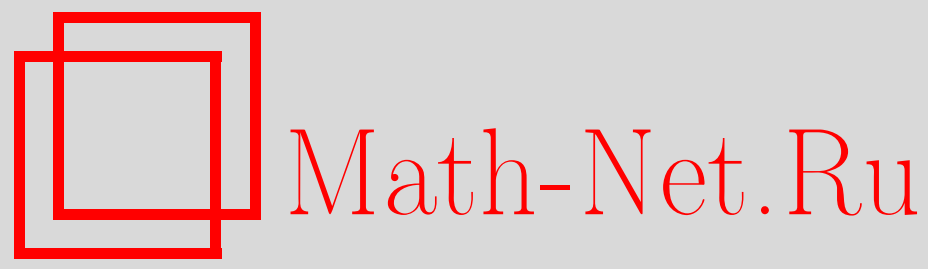

Л. Д. Эскин, Об интегральном уравнении, описывающем фазовые переходы в системе магнитных стержней, Функи. анализ и его прил., 1999, том 33, выпуск 1, 92-95

DOI: https://doi.org/10.4213/faa347

Использование Общероссийского математического портала MathNet.Ru подразумевает, что вы прочитали и согласны с пользовательским соглашением

http://www.mathnet.ru/rus/agreement

Параметры загрузки:

IP : 54.92 .164 .108

26 апреля 2023 г., 18:03:17

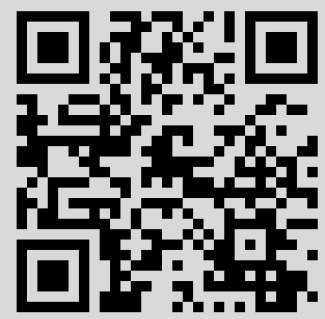


функционального анализа в математической физике. Новосибирск, СО АН СССР, 1962. 7. Сансоне Дж. Обыкновенные дифференциальные уравнения. Т. 1, ИЛ, М. 1953.

Институт математики

и механики АН Азербайджана

Поступило в редакцию

13 мая 1994

В переработанном виде

21 июля 1997 г.

УдК $517.988+517.968 .4$

\title{
Об интегральном уравнении, описывающем фазовые переходы в системе магнитных стержней^
}

\author{
(c) 1999. Л. Д. Эскин
}

1. В 1949 г. Л. Онзагер [1] показал, что в системе тонких неполярных цилиндрических стержней $(\delta=d / l \ll 1, d$ - диаметр, $l$ - длина стержня) с парным взаимодействием типа стерического отталкивания, изотропной при низких концентрациях, с увеличением концентрации происходит фазовый переход первого рода (со скачком концентрации) в анизотропную (ориентационно упорядоченную) нематическую фазу. Термодинамические свойства анизотропной фазы (нематика) полностью описываются отличной от единицы плотностью распределения ориентаций стержней $f(\mathbf{n})(\mathbf{n}-$ орт оси стержня), для которой Онзагер получил нелинейное интегральное уравнение $\nu+\ln f\left(\mathbf{n}^{\prime}\right)+\sigma T f=0$, где $\sigma=2 c d l^{2}$ (c- плотность системы), $T$ - линейный интегральный оператор, $T f=\int B_{1}\left(\mathbf{n}, \mathbf{n}^{\prime}\right) f(\mathbf{n}) d \mathbf{n}$, с ядром $B_{1}=\left(1-\left(\mathbf{n n}^{\prime}\right)^{2}\right)^{1 / 2}, \mathbf{n n}^{\prime}$ - скалярное произведение ортов $\mathbf{n}$ и $\mathbf{n}^{\prime}$, а неизвестная константа $\nu$ определяется условием нормировки $\int f(\mathbf{n}) d \mathbf{n}=1$, $d \mathbf{n}=(4 \pi)^{-1} \sin \theta d \theta d \varphi(\varphi, \theta-$ сферические координаты орта $\mathbf{n})$. Для изотропной фазы $f(\mathbf{n})=1$. Нематик обладает аксиальной и зеркальной симметрией. Направление оси симметрии совпадает с единственным направлением преимущественной ориентации осей частиц. Отсюда следует $[2,3]$, что в сферической системе координат с полярной осью, совпадающей с осью симметрии нематика, плотность $f(\mathbf{n})$ анизотропной фазы должна удовлетворять следующим дополнительным условиям: (a) $f(\mathbf{n})$ не зависит от угла $\varphi$ $(f(\mathbf{n})=f(\theta))$; (b) $f(\theta)=f(\pi-\theta)$; следовательно, $f(\mathbf{n})$ разлагается в ряд Фурье по полиномам Лежандра $P_{2 s}$ с четным индексом; (c) $f(0)=f(\pi)=\max$ и других максимумов $f$ не имеет. Решение уравнения Онзагера, удовлетворяющее условию нормировки и условиям (a)-(c), изучалось во многих физических работах (соответствуюшие ссылки можно найти в монографии [2]). Большой интерес представляет изучение системы магнитных стержней (с магнитным моментом $\mathbf{m}$ на единицу длины стержня), в которой помимо стерического отталкивания необходимо учитывать и диполь-дипольное взаимодействие

* Работа выполнена при финансовой поддержке Российского фонда фундаментальных исследований (проект 95-01-00270а). 
стержней $[4,5]$. Интегральное уравнение для плотности $f(\mathbf{n})$ в случае антиферромагнитного нематика найдено в [4]. В безразмерной форме оно имеет вид

$$
\nu+\ln f\left(\mathbf{n}^{\prime}\right)+\frac{\lambda \delta}{2 \pi \gamma} T f-\frac{\gamma \lambda}{\pi^{2}} \int \frac{B_{2}\left(\mathbf{n}, \mathbf{n}^{\prime}, \mathbf{k}, z\right) f(\mathbf{n}) d \mathbf{n} d \mathbf{k} d z}{z^{2}\left(1+2 \lambda z^{-2} \int f(\mathbf{n}) \sin ^{2}(\mathbf{k n} z / 2) d \mathbf{n}\right)}=0 .
$$

В (1) $\lambda=8 \pi c \mathbf{m}^{2} l^{2} / k_{B} T_{0}$ и $\gamma=2(\mathbf{m} l)^{2} / k_{B} T_{0} l^{3}$ - безразмерные положительные параметры, $B_{2}=\sin ^{2}(\mathbf{k n} z / 2) \sin ^{2}\left(\mathbf{k n}^{\prime} z / 2\right)$, где $\mathbf{k}$ - орт со сферическими координатами $\varphi_{0}, \theta_{0}, d \mathbf{k}=\sin \theta_{0} d \varphi_{0} d \theta_{0}$, интеграл по $\mathbf{n}$ и $\mathbf{k}$ в (1) берется по поверхности сферы, а по $z$ - от 0 до $\infty, k_{B}$ - константа Больцмана, $T_{0}$ - абсолютная температура. В случае $\mathbf{m}=0$ (стержни немагнитны) уравнение (1) совпадает с уравнением Онзагера, но оно значительно сложнее последнего. Для любых $\lambda, \gamma, \delta$ уравнение (1) при подходящем выборе неизвестной постоянной $\nu$ имеет изотропное решение $f(\mathbf{n})=1$. Анизотропные решения ответвляются от изотропного в точках бифуркации $\lambda=\lambda_{b}$. В настоящей работе с помощью идей теории Ляпунова-Шмидта изучаются близкие к изотропному анизотропные решения уравнения (1), удовлетворяющие условиям (a)-(c), т. е. описывающие нематик. Полностью описана аналитическая структура этих решений в окрестности невырожденной точки бифуркации (теорема 1), полностью исследован и вопрос об их направлении бифуркации (теорема 2).

2. Полагая $f=1+h(\mathbf{n})$ и линеаризуя уравнение $(1)$ около $h=0$, получим линейное интегральное уравнение $h-\int B\left(\mathbf{n}, \mathbf{n}^{\prime}\right) h(\mathbf{n}) d \mathbf{n}=0$, где

$$
B=-\frac{\lambda \delta}{2 \pi \gamma} B_{1}+\frac{\lambda \gamma}{\pi^{2}} \int_{0}^{\infty} \frac{\int B_{2}\left(\mathbf{n}, \mathbf{n}^{\prime}, \mathbf{k}, z\right) d \mathbf{k} d z}{z^{2}(1+\lambda \rho(z))^{2}}, \quad \rho=\frac{1}{z^{2}}\left(1-\frac{\sin z}{z}\right) .
$$

ПРЕДЛОЖЕНИЕ 1. Собственными функииями ядра В, удовлетворяюшими условиям (a) и (b), являются полиномь Лежандра $P_{2 s}$, причем

$$
\begin{gathered}
\int B\left(\mathbf{n}, \mathbf{n}^{\prime} ; \lambda\right) P_{2 s}(\mathbf{n}) d \mathbf{n}=N_{s}(\lambda, \gamma, \delta) P_{2 s}\left(\mathbf{n}^{\prime}\right), \\
N_{s}(\lambda, \gamma, \delta)=\frac{(2 s-3) ! !(2 s-1) ! ! \lambda \delta}{2^{2 s+3} s !(s+1) ! \gamma}+\frac{\lambda \gamma}{2} \int_{0}^{\infty} \frac{J_{2 s+1 / 2}^{2}(z) d z}{z^{3}(1+\lambda \rho)^{2}}, \quad s \geqslant 1
\end{gathered}
$$

$\left(J_{\nu}(z)\right.$ - бесселева функиия).

Из предложения 1 следует, что точками бифуркации уравнения (1) являются корни $\lambda_{b}(\gamma, \delta, s)$ любого из уравнений $N_{s}(\lambda, \gamma, \delta)=1$.

ОПРЕДЕЛЕНИЕ. Точку бифуркации $\lambda_{b}(\gamma, \delta, s)$ будем называть невырожденной, если она не является корнем ни одного из уравнений $N_{k}(\lambda, \gamma, \delta)=1$ с $k \neq s$. В противном случае точку бифуркации будем называть вырожденной.

ЗАмечАНИЕ 1 . Среди полиномов $P_{2 s}$ условию (c) удовлетворяет лишь $P_{2}$.

С учетом замечания 1 можно показать, что анизотропное решение, ответвляющееся от изотропного в невырожденной точке бифуркации $\lambda_{b}(\gamma, \delta, s)$, может удовлетворять условию (с) лишь в случае $s=1$. Поэтому ниже рассматриваются лишь точки бифуркации $\lambda_{b}(\gamma, \delta)=\lambda_{b}(\gamma, \delta, 1)$ и решения уравнения (1), ответвляющиеся от изотропного в этих точках. Уравнение для точек 
бифуркации $\lambda_{b}(\gamma, \delta)$ удобно записать в виде $g_{1}=g_{2}$, где

$g_{1}=\frac{\lambda}{\gamma}\left(1-\frac{\lambda \delta}{64 \gamma}\right), \quad g_{2}=\frac{\lambda^{2}}{\pi} \int_{0}^{\infty} \frac{F^{2}(z) d z}{z^{2}(1+\lambda \rho)^{2}}, \quad F=\frac{3 \sin z-3 z \cos z-z^{2} \sin z}{z^{3}}$.

Таким образом, точками бифуркации являются абсциссы точек пересечения параболы $y=g_{1}(\lambda)$ и кривой $y=g_{2}(\lambda), \lambda>0$. Кривая $g_{2}(\lambda)$ монотонно возрастает и имеет точку перегиба $\lambda=\lambda_{\text {п }}\left(\lambda_{\text {п }}\right.$ без труда находится численно).

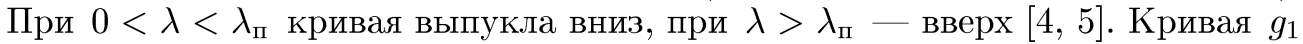
при фиксированных $\gamma, \delta$ - парабола, вершина которой при возрастании $\gamma$ перемещается вправо по прямой $y=16 / \delta$. При $\delta<\delta_{0}$ ( $\delta_{0}$ достаточно мало) и некоторых $\gamma=\gamma_{R}$ и $\gamma=\gamma_{Q}$ восходящая (левая) ветвь параболы касается выпуклой вверх части кривой $g_{2}$ сначала извне в точке $R$, а затем изнутри в точке $Q$; кроме того, при некотором $\gamma=\gamma_{P}$ вершина параболы совпадает с точкой пересечения $P$ кривой $g_{2}(\lambda)$ и прямой $y=16 / \delta$. С учетом сказанного можно показать, что существуют три непрерывные ветви точек бифуркации $\lambda_{b}(\gamma, \delta)$. Первую ветвь $\lambda_{b}^{(1)}$ образуют абсциссы точек пересечения нисходящей ветви параболы $g_{1}$ с кривой $g_{2}$. Эта ветвь определена (как функция аргумента $\gamma)$ при $0<\gamma \leqslant \gamma_{P} \sim 2^{9} / \delta$, монотонно возрастает по аргументу $\gamma$ и убывает по аргументу $\delta$, причем $32 \gamma / \delta \leqslant \lambda_{b}^{(1)}(\gamma, \delta) \leqslant \lambda_{P} \sim 2^{14} \delta^{-2}$ $(\delta \ll 1)$. Вторую ветвь образуют абсциссы лежащих левее $R$ точек пересечения восходящей ветви параболы $g_{1}$ с кривой $g_{2}$. Эта ветвь определена при $\gamma_{R}<\gamma<\infty$ и монотонно убывает по каждому из аргументов $\gamma, \delta$, причем справедлива очевидная оценка $0<\lambda_{b}^{(2)}<\lambda_{R}$ и $\lambda_{b}^{(2)} \rightarrow 0$ при $\gamma \rightarrow \infty$. Наконец, третью ветвь $\lambda_{b}^{(3)}(\gamma, \delta)$ образуют абсциссы лежащих правее $R$ точек пересечения восходящей ветви параболы $g_{1} \mathrm{c} g_{2}$. Эта ветвь определена при $\gamma_{R}<\gamma<\gamma_{Q}$ и монотонно возрастает по каждому из аргументов $\gamma, \delta$, причем $\lambda_{R}<\lambda_{b}^{(3)}<\lambda_{Q}\left(\lambda_{Q}<\lambda_{P}\right)$. Точки бифуркации $\lambda_{R}\left(\gamma_{R}, \delta\right)$ и $\lambda_{Q}\left(\gamma_{Q}, \delta\right)$ удобно рассматривать отдельно. Справедлива

Теорема 1. Пусть $\delta_{0}>0$ достаточно мало, $\delta \leqslant \delta_{0}$. Тогда решение уравнения (1), удовлетворяющее условию нормировки и условиям (a), (b) и ответвляющееся от изотропного в невырожденной точке бифуркации $\lambda_{b}(\gamma, \delta)$ разлагаетсл в окрестности этой точки в степенной ряд по чельм степеням $\mu=\lambda-\lambda_{b}$, сходящийся для достаточно мальх $|\mu|$. В окрестности точек $\lambda_{R}\left(\gamma_{R}, \delta\right)$ и $\lambda_{Q}\left(\gamma_{Q}, \delta\right)$ имеем $f(\mathbf{n})=1+\mu^{2}\left(-L_{12} / L_{20}\right) P_{2}+O\left(\mu^{3}\right), в$ окрестности остальных точек бифуркации $\lambda_{b}(\gamma, \delta)$ получаем $f(\mathbf{n})=1+$ $\mu\left(-L_{11} / L_{20}\right) P_{2}+O\left(\mu^{2}\right)$, әдe

$$
\begin{gathered}
L_{11}=\frac{\delta}{64 \gamma}+\frac{\gamma}{\pi} \int_{0}^{\infty} \frac{\left(1-\lambda_{b} \rho\right) F^{2} d z}{z^{2}\left(1+\lambda_{b} \rho\right)^{3}}, \quad L_{12}\left(\lambda_{b}, \gamma\right)=\frac{\gamma}{\pi} \int_{0}^{\infty} \frac{\left(\lambda_{b} \rho-2\right) \rho F^{2} d z}{z^{2}\left(1+\lambda_{b} \rho\right)^{3}}, \\
L_{20}=\frac{1}{7}\left(1-\frac{2 \gamma \lambda_{b}^{2}}{\pi} \int_{0}^{\infty} \frac{F^{3} d z}{z^{4}\left(1+\lambda_{b} \rho\right)^{3}}\right) .
\end{gathered}
$$

ЗАмЕчАНИЕ 2. Для построения формального разложения анизотропной плотности $f(\mathbf{n})$ в ряд по целым степеням $\mu$ следует разложить левую часть уравнения (1) в интегро-степенной ряд по $h$ и $\mu$, а затем, положив $\nu=$ 
$\sum_{k=1}^{\infty} \nu_{k} \mu^{k}, h=\sum_{k=1}^{\infty} h_{k}(\mathbf{n}) \mu^{k}$, воспользоваться методом неопределенных коэффициентов для определения $\nu_{k}$ и $h_{k}(\mathbf{n})$.

Остается выяснить, когда анизотропные решения уравнения (1), ответвляющиеся от изотропного в невырожденной точке бифуркации $\lambda_{b}(\gamma, \delta)$ и удовлетворяющие условиям (a) и (b), будут удовлетворять и условию (c). Окончательный ответ на этот вопрос дает

Теорема 2. Пусть $\delta_{0}$ достаточно мало. Тогда при любом $\delta \leqslant \delta_{0}$

1) анизотропное решение $f(\mathbf{n})$, удовлетворяющее условию нормировки и условиям (a), (b) и ответвляюшееся в любой невырожденной точке биФуркачии $\lambda_{b}(\gamma, \delta)$, принадлежащей первой или второй ветви, имеет левое направление бифуркации (т.е. удовлетворлет условию (с) лишь слева от точки бифуркачии (при $\mu<0))$;

2) наоборот, анизотропная плотность, ответвляюшаяся от изотропной в произвольной невырожденной точке третьей ветви, имеет правое направление бифуркаиии;

3) при $\gamma=\gamma_{R}$ в точке бифуркаиии $\lambda_{b}=\lambda_{R}$ ответвляется анизотропная плотность $f(\mathbf{n})$, удовлетворяющал условиям (a)-(c) в полной окрестности точки $\lambda_{R}\left(\right.$ m. е. как слева, так и справа от $\left.\lambda_{R}\right)$;

4) при $\gamma=\gamma_{Q}$ в точке бифуркачии $\lambda_{Q}$ ответвляется плотность $f(\mathbf{n})$, удовлетворяюшая условиям (a), (b), но не удовлетворяющая условию (c) ни справа, ни слева от $\lambda_{Q}$ (следовательно, и не описывающая нематик).

Ситуация, в которой, как в случаях 1) и 2) теоремы 2, ответвляющееся решение оказывается определенным лишь в полуокрестности точки бифуркации, широко распространена в теории ветвления и обычно связана с требованием вещественности решения. Однако в рассматриваемой нами задаче эта ситуация обусловлена именно требованием, чтобы анизотропное решение удовлетворяло условию (с) (т. е. описывало нематическую фазу системы). Важную роль теоремы 2 для теории фазовых переходов в системе магнитных стержней обусловливает то обстоятельство, что, как хорошо известно из физической литературы [3], именно в окрестности точки бифуркации с левым направлением бифуркации происходит фазовый переход первого рода.

\section{ЛИТЕРАТУРА}

1. Onsager L. Ann. New York Acad. Sci., 51, 627 (1949). 2. Де Жен П.-Ж. Физика жидких кристаллов. Мир, М., 1972. 3. Kayser R. F., Raveche H. J. Phys. Rev. A., 17, No. 6, 2067-2072 (1978). 4. Корнев К. Г., Эскин Л. Д. Изв. АН СССР, сер. физич., 55, вып. 6, 1050-1054 (1991). 5. Цеберс А. О. Магнитная гидродинамика, № $2,39-44$ (1983).

Казанский государственный университет

Поступило в редакцию 20 июня 1997 г. 